\title{
The Method of the SiC MOSFET Replacing the Si IGBT in the Traditional Power Electronics Converter without Redesigning the Main Circuit and the Driver Circuit
}

\author{
Lei Zhang*, Dejian Yang, Lei Ren, Yun Cheng, Qiufeng Yan and Yinlong Yuan \\ School of Electrical Engineering, Nantong University, Nantong, 226019, China \\ *Corresponding Author: Lei Zhang. Email: nttzzl@ntu.edu.cn \\ Received: 07 October 2020 Accepted: 02 December 2020
}

\begin{abstract}
As a wide bandgap power electronics device, the $\mathrm{SiC}$ MOSFET has a lot of advantages over the traditional $\mathrm{Si}$ IGBT. Replacing the Si IGBT with the SiC MOSFET in the existing power electronics converter is an effective means to improve the performance and promote the upgrading of the traditional converter. Generally, in order to make full use of its advantages of the SiC MOSFET, the Si IGBT in the traditional power electronics circuit cannot be simply replaced by the SiC MOSFET, but the main circuit and the driver circuit need to be redesigned because the oscillation problem and the cross-talk problem caused by the parasitic parameter are very serious when the SiC MOSFET switches. However, considering the low cost and the short cycle requirement of the equipment development, it is often difficult to redesign the circuit hardware structure of all converters. In order to resolve this contradiction, a ferrite bead based method is proposed in this paper. Through this method, without redesigning the hardware structure of the main circuit and the driver circuit, the SiC MOSFET can be used to replace the Si-based power electronics device directly, and the loss of the converter can be reduced with small oscillation. Finally, the effectiveness of the proposed method is proved by experiments.
\end{abstract}

\section{KEYWORDS}

SiC MOSFET; oscillation; cross-talk; parasitic parameter; ferrite bead

\section{Introduction}

Nowadays, the SiC MOSFET has received extensive attention for its excellent performance in the switching speed, the switching frequency, the rated voltage value, the on-resistance, the radiation resistance, the temperature resistance and other aspects [1-5]. The application of the SiC MOSFET in the converter can effectively improve the system efficiency and promote the system upgrading [6-10]. However, the SiC MOSFET also faces several crucial problems. For example, the fast switching speed of the SiC MOSFET makes it very sensitive to the parasitic parameters of the main circuit and the driver circuit, which may produce huge oscillations and cause the system to collapse $[11,12]$. This makes it impossible to directly replace the Si IGBT in the traditional converter without redesigning the circuit.

To solve the aforementioned problems, a large number of literatures have studied the oscillation principle and the low parasitic parameter circuit of the SiC MOSFET converter. Ke et al. [13] used the simulation model to analyze the relationship between the SiC MOSFET oscillation and the parasitic

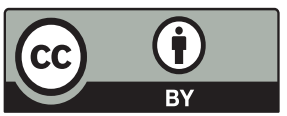

This work is licensed under a Creative Commons Attribution 4.0 International License, which permits unrestricted use, distribution, and reproduction in any medium, provided the original work is properly cited. 
parameter during the turn-off process, but no practical solutions are given. Cree/Wolfspeed [14] found that the parasitic inductance of the main circuit can cause the huge oscillation and suggested that the DC bus-bar of the SiC MOSFET converter should adopt a parallel array of small capacitors instead of one large capacitor to reduce the parasitic inductance introduced by the DC capacitor, and the magnetic field cancelling technology should be adopted to reduce the line parasitic inductance and further reduce the DC capacitor parasitic inductance. Li et al. [15] demonstrated that parasitic capacitance of the inductor load in the $\mathrm{SiC}$ MOSFET converter and the common source inductance of the SiC MOSFET can cause oscillation of the circuit, so it is recommended that the single layer of windings of the inductor and Quasi-Kelvin-Source connection of the SiC MOSFET should be used. Huang et al. [16] demonstrated the switching ringing mechanism of the SiC MOSFET converter and proposed an active gate drive to suppress the circuit oscillation. Kim et al. [17] modeled the switching circuit of the SiC MOSFET converter considering the parasitic components and proposed an air core PCB transformer with a properly designed secondary side circuit inserted into the main circuit to dampen the oscillation. Chen et al. [18], Yatsugi et al. [19], Chuai et al. [20] and Liu et al. [21] also analyzed the huge oscillation problem of the whole circuit after the adoption of the SiC MOSFET and suppressed it by adding the RC snubber circuit in the main circuit. In Chen et al. [18], the RC circuit was added between the DC bus-bar to dampen the oscillation and the corresponding parameters of the RC circuit were selected by the trial and error empirical method. Yatsugi et al. [19] also adopted the method of the parallel RC circuit at the bus-bar to suppress the oscillation caused by the parasitic inductance of the bus, but it used the root locus method to calculate the parameters of the RC circuit, which made the parameters more universal. Chuai et al. [20] analyzed the parasitic inductance in the circuit through the ANSYS Q3D software, and then designed the corresponding RC circuit to suppress the oscillation generated by the inductance. Through model analysis and formula derivation, Liu et al. [21] proposed a better design of the bus-bar structure and parallel connection of the RC snubber, which decreased the stray inductance of the bus-bar by $93.6 \%$ and the total loop-inductance by $46.4 \%$. Yatsugi et al. [22] designed an external RLC resonator through analogy with passive parity-time (PT) symmetry, which was implemented in parallel to a bus-bar and has a better ringing suppression effect. Although these methods mentioned above are useful for better use of the $\mathrm{SiC}$ MOSFET, they all require modifying the main circuit or designing a driver circuit to suppress oscillation. These circuit modifications require a lot of time and cost, which is not conducive to the development from the $\mathrm{Si}$ based converter products to the $\mathrm{SiC}$ MOSFET converter products.

Besides the oscillation problem mentioned above, it also presents a serious cross-talk problem, which is exacerbated when the main circuit oscillates because of the SiC MOSFET's extremely fast switching speed. The cross-talk is a hot issue and its mechanism and suppression method have been studied in a large number of literatures. Jahdi et al. [23] conducted the detailed analysis of the cross-talk mechanism in the SiC MOSFET circuit and the Si IGBT circuit. Through the modeling and the experimental comparison, it is found that the cross-talk in the $\mathrm{SiC}$ MOSFET circuit is much more serious than that in the Si IGBT circuit. For the cross-talk problem, Zhang et al. [24] compared the hazardous degree when the SiC MOSFET turns on and turns off on the situation of the parasitic inductance, and found that the cross-talk problem when the SiC MOSFET turns off is more serious. In order to provide the theoretical basis for the cross-talk suppression, Zhang et al. [25] and Xu et al. [26] analyzed the influence of the driver resistance and the driver capacitance on the cross-talk. Li et. al. [27] considered the nonlinearity of gate-drain capacitance and proposed a predictive algorithm for accurately determining the cross-talk, which was proved to be more accurate by experiments. Yin et al. [28], Zhang et al. [29], Tang et al. [30], Li et al. [31] and Zhang et al. [32] proposed several measures to suppress the SiC MOSFET cross-talk, including the turn-on and turn-off resistance separation, increasing the gate-source capacitance, the miller capacitance active clamp and the gate auxiliary circuit. These methods all require a large modification of the whole circuit. 
For upgrading the traditional converters by replacing the Si IGBT with the SiC MOSFET, it is often necessary to redesign the entire circuit hardware structure to decrease the huge oscillation and the crosstalk caused by the fast switching speed of the SiC MOSFET, which needs large development cycle and cost. Therefore, this paper proposes a solution of inserting the ferrite bead in the main circuit at a certain position to suppress the oscillation, so that the SiC MOSFET can directly replace the Si IGBT to upgrade the traditional converter without redesigning the circuit hardware. This is a new scenarios and application areas and the purpose of this paper is to provide a fast and low-cost converter upgrading scheme based on the traditional platform for converter manufacturers.

The structure of the paper is as follows. Section 2 introduces the working states of the SiC MOSFET in the main circuit suitable for the Si IGBT. The function of the ferrite bead in the main circuit is analyzed in Section 3 and Section 4 provides the experiment results. Section 5 concludes the paper.

\section{Working States of the SiC MOSFET in the Main Circuit Suitable for the Si IGBT}

In order to show the oscillation problem of directly applying the SiC MOSFET to the main circuit, this section takes a double-pulse test (DPT) circuit as an example to analyze the turn-on and turn-off processes of the SiC MOSFET in this DPT circuit.

\subsection{DPT Circuit}

The schematic of the DPT circuit is shown in Fig. $1 . V$ is the adjustable DC voltage source. $C_{1}$ is the DC electrolytic capacitor and $C_{2}$ is the DC film capacitor. $\mathrm{M}_{1}$ and $\mathrm{M}_{2}$ are the SiC MOSFETs, and $\mathrm{D}_{1}$ and $\mathrm{D}_{2}$ are their corresponding body diodes. $L$ is the inductor load and $R$ is its resistance. $v_{\mathrm{G}}$ is the driver voltage of the $\mathrm{M}_{2} . R_{\mathrm{G} 1}$ and $R_{\mathrm{G} 2}$ are the driver resistors. $L_{\mathrm{dc}}, L_{\mathrm{dcr}}, L_{\mathrm{D} 1}, L_{\mathrm{D} 2}, L_{\mathrm{S} \_ \text {COM1}}, L_{\mathrm{S} \_\mathrm{COM} 2}, L_{\mathrm{G} 1}$ and $L_{\mathrm{G} 2}$ are the parasitic inductances of the main circuit and the devices, which can be introduced detailly: $L_{\mathrm{dc}}$ and $L_{\mathrm{dcr}}$ are the parasitic inductances of the positive and negative DC bus-bar respectively; $L_{\mathrm{D} 1}$ and $L_{\mathrm{D} 2}$ are the drain inductances; $L_{\mathrm{S}_{-} \mathrm{COM} 1}$ and $L_{\mathrm{S}_{-} \mathrm{COM} 2}$ are the common source inductances; $L_{\mathrm{G} 1}$ and $L_{\mathrm{G} 2}$ are the gate inductances. $C_{\mathrm{GD} 1}, C_{\mathrm{GD} 2}, C_{\mathrm{GS} 1}, C_{\mathrm{GS} 2}, C_{\mathrm{DS} 1}, C_{\mathrm{DS} 2}, C_{\mathrm{L}}$ are the parasitic capacitances of the devices and the load, which can be introduced detailly: $C_{\mathrm{GD} 1}$ and $C_{\mathrm{GD} 2}$ are the Miller capacitances; $C_{\mathrm{GS} 1}$ and $C_{\mathrm{GS} 2}$ are the gate-source capacitances; $C_{\mathrm{DS} 1}$ and $C_{\mathrm{DS} 2}$ are the drain-source capacitances; $C_{\mathrm{L}}$ is the parasitic capacitance of the inductor load. The driver voltage of $\mathrm{M}_{1}$ is $-5 \mathrm{~V}$, which means the channel of $\mathrm{M}_{1}$ is off and only the body diode works.

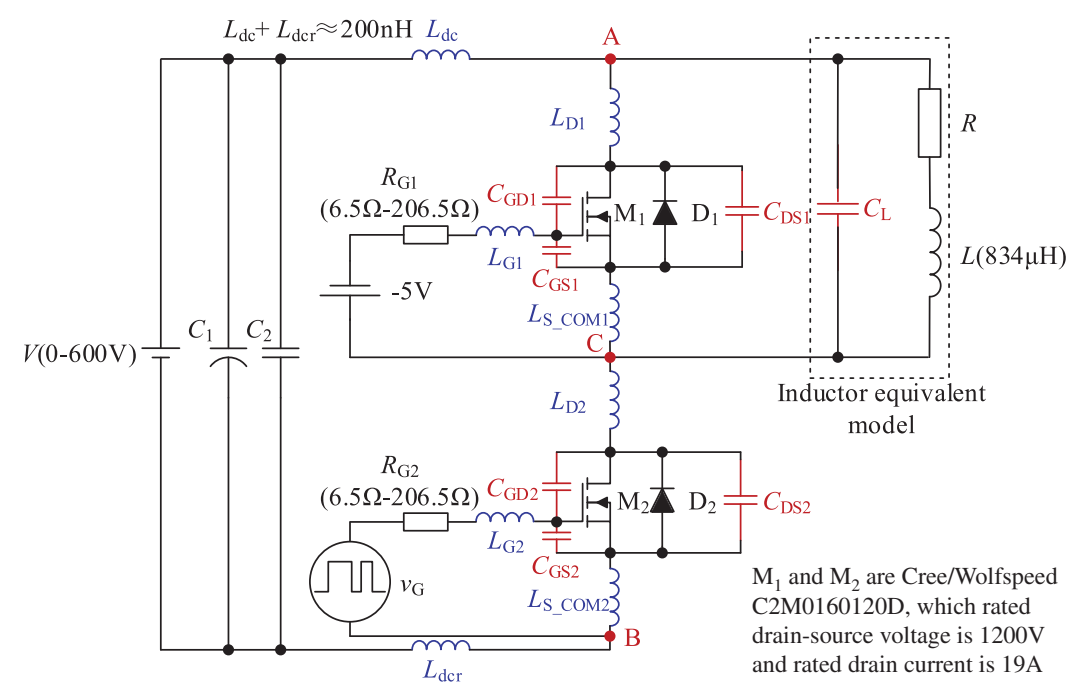

Figure 1: Schematic diagram of the DPT circuit 
The driver voltage $v_{\mathrm{G}}$ is a double-pulse signal, which is shown in Fig. 2. The low voltage of the doublepulse signal is about $-5 \mathrm{~V}$ to ensure the SiC MOSFET turned off reliably. The high voltage of the double-pulse signal often exceeds $20 \mathrm{~V}$ to make the SiC MOSFET turned on fully. At the falling edge of the first pulse, the circuit state when the SiC MOSFET $\mathrm{M}_{2}$ turns off can be tested. At the rising edge of the second pulse, the circuit state when the SiC MOSFET $\mathrm{M}_{2}$ turns on can be tested.

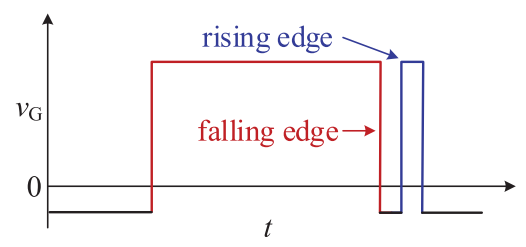

Figure 2: Double pulse signal

\subsection{Circuit Analysis When $M_{2}$ Turns on}

When $\mathrm{M}_{2}$ is on a stable off-state or a stable on-state, the circuit can operate steadily no matter how large the parasitic inductance in the circuit is. These processes are the same as those of the Si IGBT converter.

When $\mathrm{M}_{2}$ is turned on, the equivalent circuit model at this time is shown in Fig. 3. The $C_{\text {oss } 1}$ is the output capacitance of $\mathrm{M}_{1}$ and $C_{\mathrm{oss} 2}$ is the output capacitance of $\mathrm{M}_{2}$. Note that the value of $C_{\mathrm{oss} 1}$ is the sum of $C_{\mathrm{DS} 1}$ and $C_{\mathrm{GD} 1}$ and the value of $C_{\mathrm{oss} 2}$ is the sum of $C_{\mathrm{DS} 2}$ and $C_{\mathrm{GD} 2} . R_{\mathrm{on}}$ is the equivalent variable resistance of $\mathrm{M}_{2}$ when it switches.

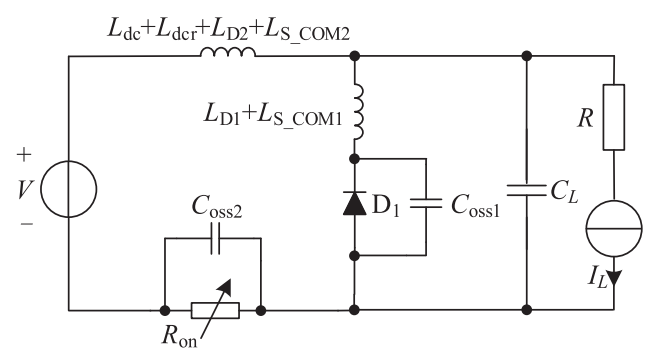

Figure 3: Equivalent model of the current flow path at the falling edge of the first pulse or at the rising edge of the second pulse

Because the switch speed of the SiC MOSFET is extremely fast, the series circuit of the DC voltage source $V$ and the variable resistance $R_{\text {on }}$ can be equivalent to a step voltage source $V_{\mathrm{s}}$. The equivalent model in Fig. 3 can be simplified as shown in Fig. 4a. According to the superposition theorem, the circuit can be disassembled into two sub circuits, which are shown in Figs. $4 \mathrm{~b}$ and $4 \mathrm{c}$, respectively.

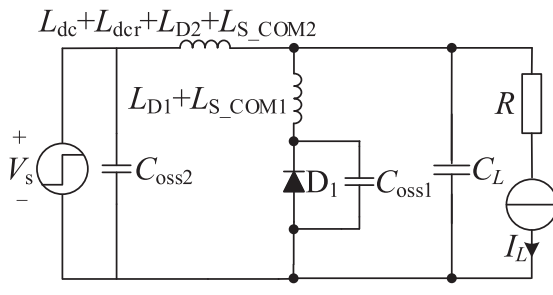

(a)

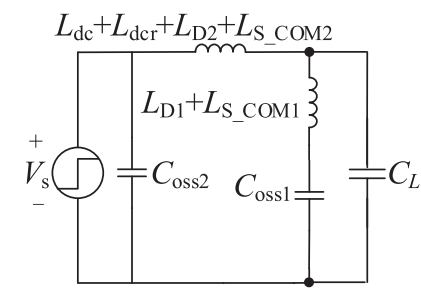

(b)

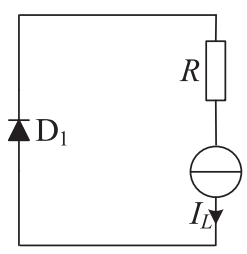

(c)

Figure 4: Simplified model of the current flow path when $\mathrm{M}_{2}$ switches: (a) simplified model, (b) considering only the voltage source, and (c) considering only the current source 
From Fig. 4, when the oscillation is caused by the equivalent step voltage, the parasitic inductance and the output capacitance will occur in the circuit. The voltage across $C_{\mathrm{oss} 1}$ can be calculated by

$V_{\text {Coss } 1}=V-V \cos \left(\frac{1}{\sqrt{L_{\text {total }} C_{\mathrm{oss} 1}}} t\right)$

where $L_{\text {total }}$ is the sum of $L_{\mathrm{dc}}, L_{\mathrm{dcr}}, L_{\mathrm{D} 1}, L_{\mathrm{D} 2}, L_{\mathrm{S} \_ \text {COM1 }}$ and $L_{\mathrm{S} \_\mathrm{COM} 2}$. This means that due to the extremely fast switching speed of the SiC MOSFET, the oscillation amplitude will reach twice the DC bus-bar voltage. Note that the oscillation will be very small when the Si IGBT is used because its switching speed is very slow and $V$ s can only be regarded as a ramp signal not a step signal.

Moreover, during the SiC MOSFET turning on, there is not only oscillation problem but also cross-talk problem, which can be explained by Fig. $5 \mathrm{a}$. When the SiC MOSFET $\mathrm{M}_{2}$ turns on, the drain-source voltage across $\mathrm{M}_{1}$ increases rapidly and the current $i_{\text {Miller }}$ through the Miller capacitance $C_{\mathrm{GD} 1}$ will be generated. The current $i_{\text {Miller }}$ will induce a positive voltage on the gate of $\mathrm{M}_{1}$. As the switching speed of $\mathrm{M}_{2}$ gradually increases, the $i_{\text {Miller }}$ will be large and then the gate voltage of $\mathrm{M}_{1}$ may exceed the threshold value. $\mathrm{M}_{1}$ will turn on falsely and the shoot-through failure will happen.

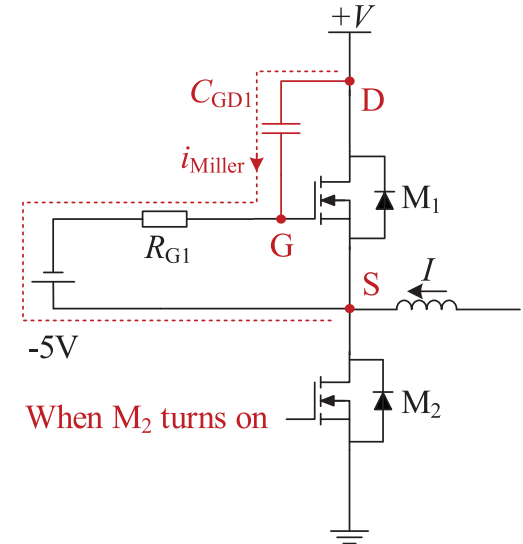

(a)

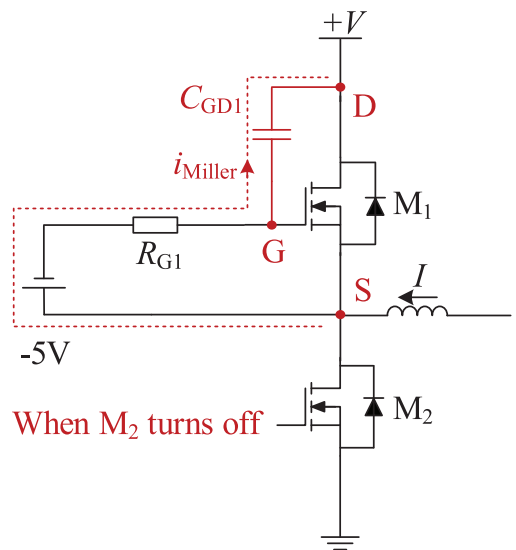

(b)

Figure 5: Cross-talk effect when $M_{1}$ is OFF: (a) when $M_{2}$ turns on, and (b) when $M_{2}$ turns off [22]

Considering the oscillation problem mentioned above, $i_{\text {Miller }}$ will contain a huge oscillation so the gate voltage will have a very serious oscillation problem.

\subsection{Circuit Analysis When $M_{2}$ Turns off}

When $\mathrm{M}_{2}$ is turned off, the equivalent circuit is similar to that when it is turned on, which is shown in Figs. $4 \mathrm{~b}$ and $4 \mathrm{c}$. The difference is that the step signal is the negative step ( $V$ to 0$)$ at this time. The voltage across $C_{\mathrm{oss} 1}$ when $\mathrm{M}_{2}$ turns off can be calculated by

$$
V_{\text {Coss } 1}^{\prime}=V \cos \left(\frac{1}{\sqrt{L_{\text {total }} C_{\text {oss } 1}}} t\right) \text {. }
$$

It means that the oscillations in the circuit will still exist when $\mathrm{M}_{2}$ turns off but the oscillation range is between $-V$ to $V$. Similarly, the oscillations here will be much larger than those in the Si IGBT converter.

Moreover, during the turning off process of the $\mathrm{M}_{2}$, there is also cross-talk problem, which can be explained by Fig. $5 b$. When $\mathrm{M}_{2}$ turns off, the drain-source voltage across $\mathrm{M}_{1}$ decreases rapidly and the current $i_{\text {Miller }}$ through the Miller capacitance $C_{\mathrm{GD} 1}$ will be generated. The current $i_{\text {Miller }}$ will induce a 
negative voltage on the gate of $\mathrm{M}_{1}$. The introduced negative voltage needs to be constrained, otherwise there is a risk of breaking through the gate oxide of $\mathrm{M}_{1}$. Compared to the turn-on process, there is no shoot-through problem and the severity is smaller to some extent.

\section{Function of the Ferrite Bead in the Main Circuit}

To replace the Si IGBT with the SiC MOSFET directly in the traditional power electronics converter, the problems of the huge oscillation and the cross-talk mentioned above must be solved. This paper presents a method of using the ferrite bead to solve the above problems and the effect of the ferrite bead is analyzed following.

\subsection{Model of the Ferrite Bead}

The ferrite bead is essentially a single-coil inductor which has great damping effect at a certain frequency range. The equivalent model of the ferrite bead is shown in Fig. 6. $L_{\mathrm{FB}}$ is the equivalent inductance of the ferrite bead. $C_{\mathrm{FB}}$ is the equivalent capacitance of the ferrite bead. $R_{\mathrm{FB} 1}$ and $R_{\mathrm{FB} 2}$ are the equivalent resistances of the ferrite bead.

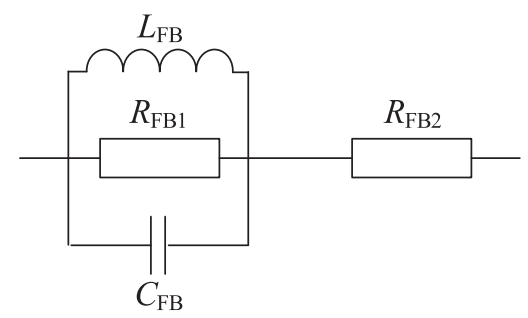

Figure 6: Equivalent model of the ferrite bead

The impedance expression of the ferrite bead can be given by

$Z_{\mathrm{FB}}=\frac{D \omega^{4}+E \omega^{2}+F+j\left(G \omega^{3}+H\right)}{A \omega^{4}+B \omega^{2}+C}$

where $\omega$ is the angular frequency and the other parameters can be expressed by

$$
\left\{\begin{array}{l}
A=R_{\mathrm{FB} 1}^{2} L_{\mathrm{FB}}^{2} C_{\mathrm{FB}}^{2} \\
B=L_{\mathrm{FB}}^{2}-2 R_{\mathrm{FB} 1}^{2} L_{\mathrm{FB}} C_{\mathrm{FB}} \\
C=R_{\mathrm{FB} 1}{ }^{2} \\
D=R_{\mathrm{FB} 2} L_{\mathrm{FB}}{ }^{2} C_{\mathrm{FB}}{ }^{2} \\
E=R_{\mathrm{FB} 1} L_{\mathrm{FB}}^{2}+R_{\mathrm{FB} 2} L_{\mathrm{FB}}{ }^{2}-2 R_{\mathrm{FB} 1}^{2} R_{\mathrm{FB} 2} L_{\mathrm{FB}} C_{\mathrm{FB}} \\
F=R_{\mathrm{FB} 1} R_{\mathrm{FB} 2} \\
G=-R_{\mathrm{FB} 1}^{2} L_{\mathrm{FB}}^{2} C_{\mathrm{FB}} \\
H=R_{\mathrm{FB} 1}{ }^{2} L_{\mathrm{FB}}^{2}
\end{array}\right.
$$

From the formula, it can be found that the ferrite bead can finally be equivalent to a series circuit of the inductance and the resistance, and the equivalent resistance and the inductance vary with the change of the frequency.

Fig. 7 gives the measured impedance characteristic curves of a common ferrite bead. The total inductance of the ferrite bead decreases with the increase of the frequency, and the total resistance of the ferrite bead increases with the increase of the frequency. 


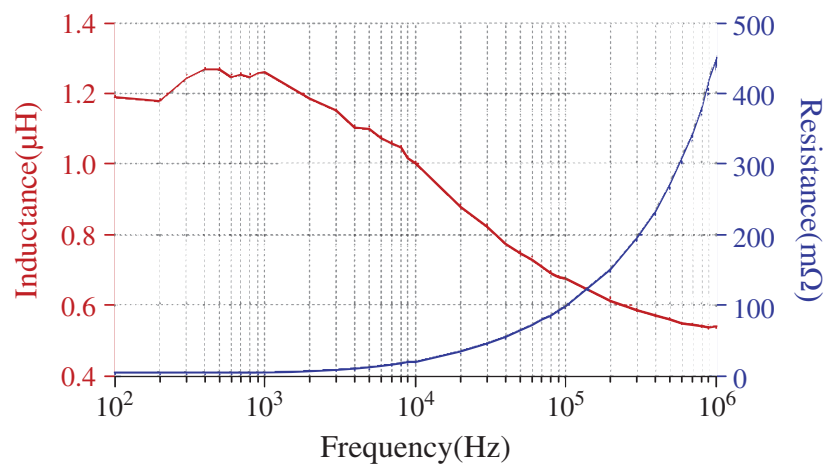

Figure 7: Measured impedance characteristic curves of a common ferrite bead

The analysis above means that the ferrite bead exhibits the inductance characteristic at the low frequency and the resistance characteristic at the high frequency. This property of the ferrite bead can be used to dampen the large high frequency oscillations of the circuit mentioned in Section 2 and has little effect on the normal switch waveforms during the SiC MOSFET switching.

\subsection{Inhibition of Oscillation by the Ferrite Bead}

To suppress the main circuit oscillation during the switching process of $\mathrm{M}_{2}$, the ferrite bead can be added in the main circuit, which is shown in Fig. 8, where FB is the added ferrite bead.

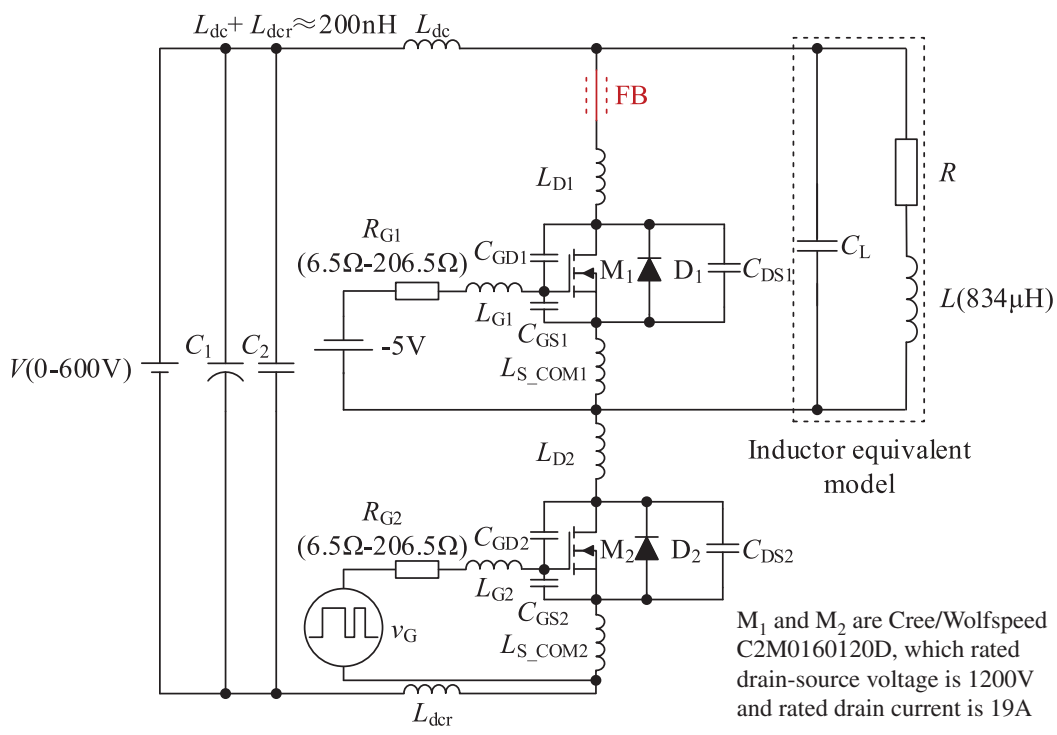

Figure 8: Ferrite bead position when $\mathrm{M}_{2}$ switched

After the ferrite bead is inserted into the main circuit, it is necessary to analyze the non-oscillation component and the oscillation component respectively. For the non-oscillation component, the inductance in the circuit when $\mathrm{M}_{2}$ turns on can be considered as a wire and the ferrite bead can be equivalent to a wire too. The simplified circuit model considering only the voltage source at this time can be shown in Fig. 9a, in which case the ferrite bead has no effect on the main circuit.

For the oscillation component, the ferrite bead can be equivalent to a resistance. The simplified circuit model considering only the voltage source at this time can be shown in Fig. $9 \mathrm{~b}$. When the $\mathrm{M}_{2}$ turns on, the 
voltage across $C_{\mathrm{oss} 1}$ is a damped or the overdamped oscillation waveform. If it is a damped oscillation, the overshoot is

$\sigma \%=e^{-\pi \xi / \sqrt{1-\xi^{2}}} \times 100 \%$

where

$\xi=\frac{R_{\mathrm{FB}}}{2} \sqrt{\frac{C_{\mathrm{oss} 1}}{L_{\mathrm{total}}}}$

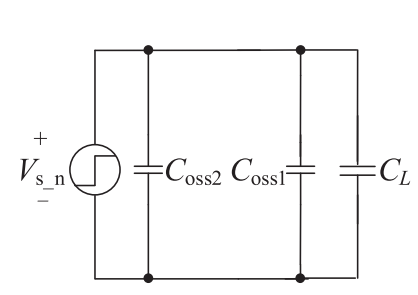

(a)

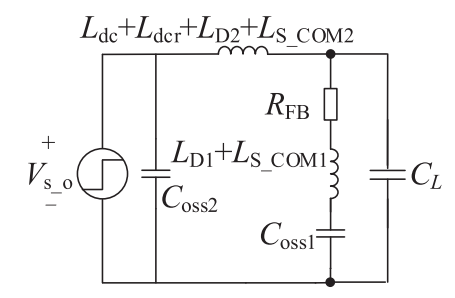

(b)

Figure 9: Simplified model considering only the voltage source: (a) for the non-oscillation component, and (b) for the oscillation component

Because of the resistance of the ferrite bead at the oscillation frequency, the oscillation in the equipment circuit can be effectively suppressed when the Si IGBT is replaced by the SiC MOSFET.

Note that when $\mathrm{M}_{2}$ is turned off, the current load will be in the ferrite bead, which will saturate the ferrite bead, in which case the ferrite bead has no influence on the oscillation problem during the turning off process in the DPT circuit. However, in practical converters, the ferrite beads can be added to the upper and the lower SiC MOSFETs of the half bridge, and the oscillation can be suppressed by the ferrite bead in series with the complementary device when it turns off.

\subsection{Inhibition of Cross-Talk by the Ferrite Bead}

The cross-talk is influenced by the ferrite bead, which is discussed as follows:

When $\mathrm{M}_{2}$ turns on, the cross-talk after the ferrite bead being inserted can be shown in Fig. 10a. Since the ferrite bead can be equivalent to a series circuit of resistance and inductance, the ferrite bead will reduce the Miller current $i_{\text {Miller }}$ and the value of gate-source voltage of $\mathrm{M}_{1}$. Therefore, the shoot-through risk will be reduced greatly. Note that when $\mathrm{M}_{2}$ turns on, the current will completely convert from $\mathrm{M}_{1}$ to $\mathrm{M}_{2}$, and then the drain-source voltage of $\mathrm{M}_{1}$ will rise, thus there is no saturation problem of the ferrite bead at this cross-talk process.

When $\mathrm{M}_{2}$ turns off, the cross-talk after the ferrite bead being inserted can be shown in Fig. 10b. The ferrite bead will reduce the Miller current $i_{\text {Miller }}$ and the gate-source voltage of $\mathrm{M}_{1}$. Therefore, the risk of breaking through the gate oxide will be reduced. Note that when the $\mathrm{M}_{2}$ turns off, the drain-source voltage of $M_{1}$ will fall firstly, and the current will convert from $M_{2}$ to $M_{1}$, thus there is no saturation problem of the ferrite bead at this cross-talk process.

\section{Experiment Result}

\subsection{Experimental Platform}

The picture of the double-pulse test platform is shown in Fig. 11. The schematic diagram is shown in Figs. 1 and 8, and the position of the ferrite bead is shown by the FB in Fig. 8. The parameters of devices are listed in the two figures. About the installation method of the ferrite bead, it is directly 
welded to the drain pin of the SiC MOSFET $\mathrm{M}_{1}$, and both of them in combination are welded to the circuit board. In the experimental platform, the C2M0160120D from Cree/Wolfspeed is chosen as the SiC MOSFET $\mathrm{M}_{1}$ and $\mathrm{M}_{2}$, whose rated voltage is $1200 \mathrm{~V}$ and rated current is $19 \mathrm{~A}$. The DC voltage source can be adjusted from $0 \mathrm{~V}$ to $600 \mathrm{~V}$. The load is the self-made air-core inductor, which can avoid the saturated load inductance. The inductance value of it is $834 \mu \mathrm{H}$. Because the experiment is to demonstrate the application of SiC MOSFETs in the main circuit which is suitable to Si IGBTs, the parasitic inductance of the main circuit $L_{\mathrm{dc}}+L_{\mathrm{dcr}}$ approximates $200 \mathrm{nH}$. Considering that the internal drive resistance of $\mathrm{M}_{1}$ and $\mathrm{M}_{2}$ is $6.5 \Omega$, the adjustment ranges of the total driver resistor $R_{\mathrm{G} 1}$ and $R_{\mathrm{G} 2}$ are $6.5 \Omega$ to $206.5 \Omega$. Due to the fast switching speed of the SiC MOSFET and the high oscillation frequency of the circuit, the bandwidth of the oscilloscope, the voltage probes and the current probe are $100 \mathrm{MHz}, 100 \mathrm{MHz}$ and $120 \mathrm{MHz}$ respectively. Further, in order to use more points to represent the ns level waveform, the sampling rate of the selected oscilloscope is $2.5 \mathrm{GS} / \mathrm{s}$.

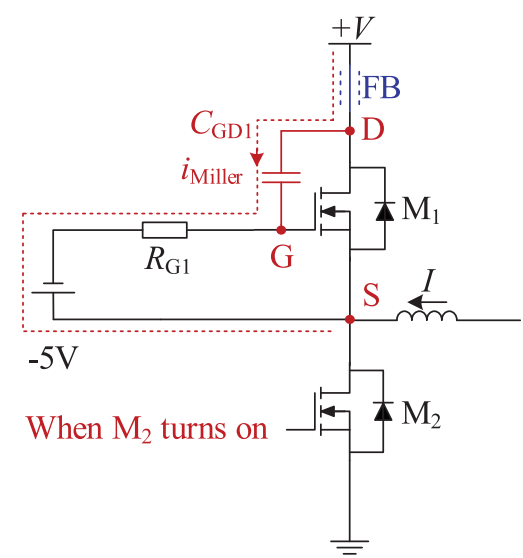

(a)

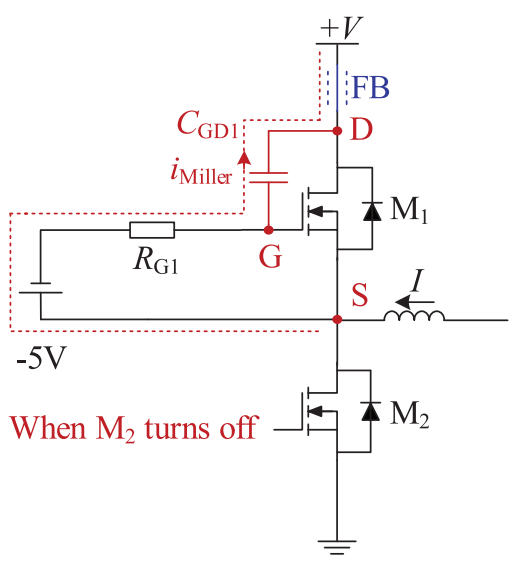

(b)

Figure 10: Cross-talk effect after the ferrite bead inserted: (a) when $M_{2}$ turns on, and (b) when $M_{2}$ turns off

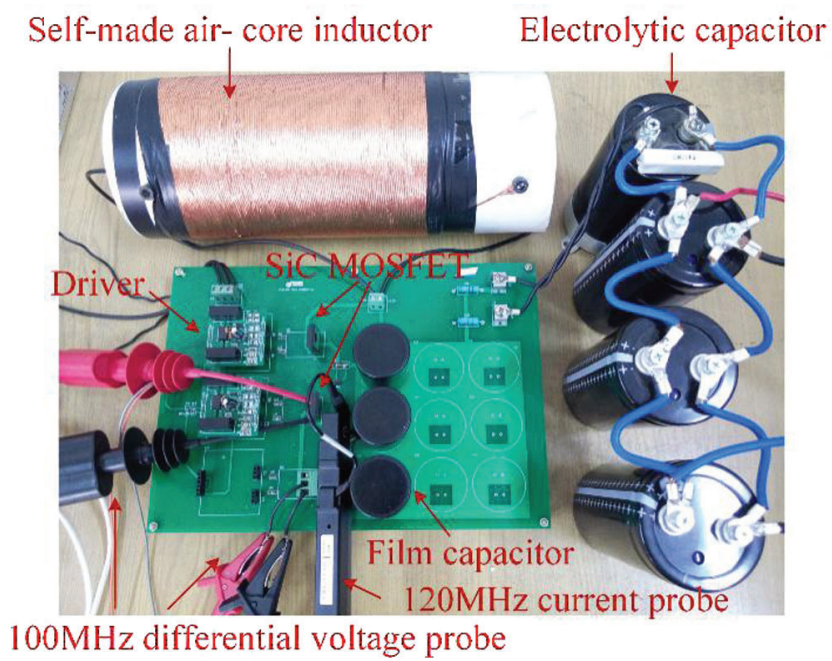

Figure 11: Experimental platform 
The traditional Si IGBT converter often has large parasitic parameters in the hardware, which is also the reason why the converter cannot work when the Si IGBT is directly replaced by the SiC MOSFET. In order to study this problem, the experimental platform in this paper is designed to simulate the situation with the large parasitic inductance in the traditional Si IGBT converter. Using the method proposed in this paper, the Si IGBT can be replaced by the SiC MOSFET without redesigning the circuit of the traditional Si IGBT converter to improve the efficiency of the converter.

\subsection{Oscillation Waveform without the Ferrite Bead}

Due to the huge parasitic inductance in the main circuit when the Si IGBT was directly replaced by the $\mathrm{SiC}$ MOSFET, the voltage and the current in the circuit will oscillate seriously. Due to the influence of the shoot-through, the oscillations will be more serious when the SiC MOSFET turns on. When the DC voltage source is $88 \mathrm{~V}$ and the driver resistance is $26.5 \Omega$ in the experiment, the huge oscillation phenomena will occur in the circuit. The waveforms of the DC bus-bar voltage, the driver supply voltage, the driver input signal (the controller output signal), the drain voltage, the drain current and the gate-source voltage of the $\mathrm{M}_{2}$ are shown in the Fig. 12. In the Figure, $V$ is the DC bus-bar voltage of the double-pulse test platform; $V_{\text {driver_supply }}$ is the driver supply voltage of $\mathrm{M}_{2} ; V_{\text {signal }}$ is the driver input signal of $\mathrm{M}_{2}$ and it is also the controller output signal for $\mathrm{M}_{2} ; V_{\mathrm{DS}}$ is the drain-source voltage of $\mathrm{M}_{2} ; i_{\mathrm{D}}$ is the drain current of $\mathrm{M}_{2} ; v_{\mathrm{GS}}$ is the gate-source voltage of $\mathrm{M}_{2}$. Note that the meanings of these symbols apply to all the figures below.

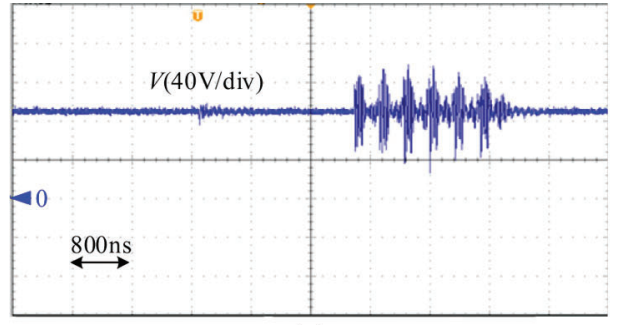

(a)

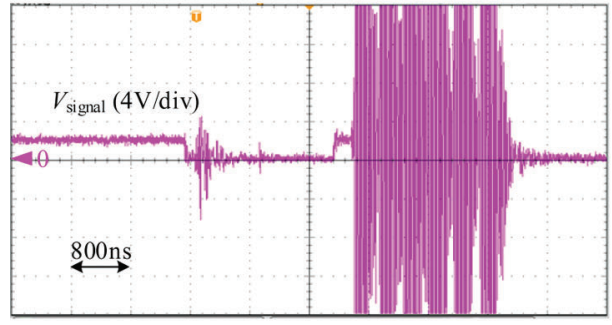

(c)

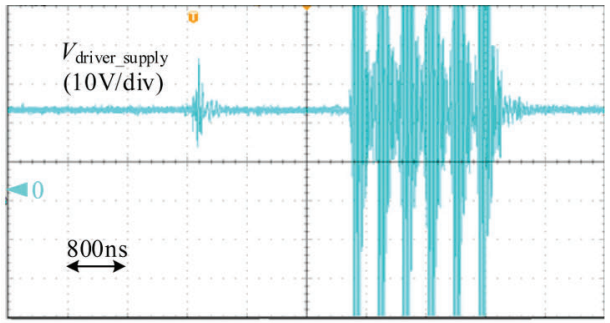

(b)

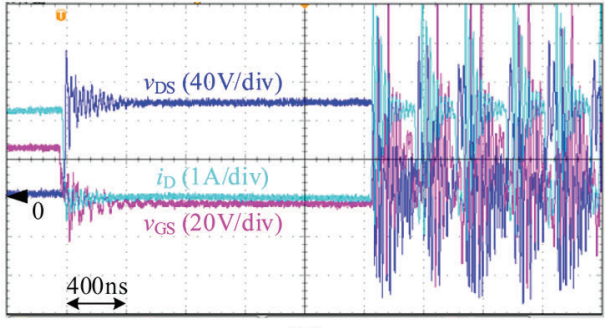

(d)

Figure 12: Oscillation waveform without the ferrite bead: (a) DC bus-bar voltage, (b) driver supply voltage, (c) driver input signal (the controller output signal), and (d) drain-source voltage, the drain current and the gate-source voltage of the $\mathrm{M}_{2}$

It can be seen from the Fig. 12 that the circuit can no longer work normally, and the corresponding waveforms are consistent with the above analysis. Due to the parasitic oscillation of the main circuit, the driver supply voltage and the controller output signal are also subjected to a huge EMI effect. Thus, the circuit will not work when the $\mathrm{SiC}$ MOSFET is used directly without taking any action. Note that the oscillation problem is much more serious when $\mathrm{M}_{2}$ is turned on than when it is turned off. This is because the shoot-through problem caused by the cross-talk when $\mathrm{M}_{2}$ is turned on will further aggravate the oscillation. 
The oscillation can be reduced by lowering the DC bus-bar voltage. When the DC bus-bar voltage drops to $28 \mathrm{~V}$, although the waveforms still oscillate, the oscillation can be attenuated and the circuit can work normally, as shown in Fig. 13. This is because when the DC bus-bar voltage decreases, the output capacitance of $\mathrm{M}_{2}$ will increase and its switching speed will slow down. $V_{\mathrm{s}}$ in the above analysis cannot be equivalent to a step signal, but can only be equivalent to a ramp signal, so the oscillation amplitude decreases. Moreover, in the situation of the reduced DC bus voltage, the shoot-through energy decreases when the cross-talk occurs, and the negative impact of the shoot-through problem on the oscillation is relieved.

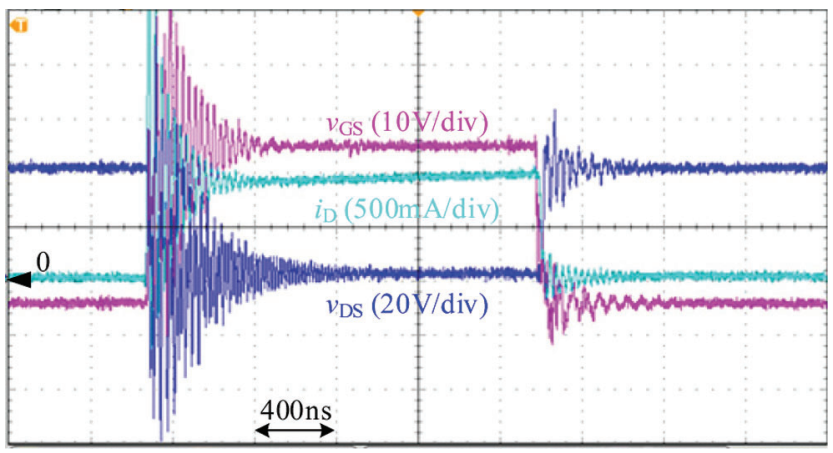

Figure 13: Waveform of $\mathrm{M}_{2}$ when the DC bus-bar voltage is low

Certainly, it is not desirable to operate a $1200 \mathrm{~V} \mathrm{SiC} \mathrm{MOSFET} \mathrm{at} \mathrm{the} 28 \mathrm{~V}$ DC bus-bar voltage, and it does not satisfy the use requirement of the SiC MOSFET in the traditional Si IGBT converter. Thus, a method must be found for the circuit to work at the high DC bus-bar voltage.

\subsection{Oscillation Suppression Method of Regulating the Driver Resistance}

According to the above analysis, when the switching speed of the SiC MOSFET decreases, the oscillation problem will be alleviated. The switching speed of the SiC MOSFET can be reduced by increasing the driver resistance. In order to make the circuit work properly at the DC bus-bar voltage of $600 \mathrm{~V}$, the driver resistance of $\mathrm{M}_{2}$ is set as $206.5 \Omega$. The drain-source voltage, the drain current and the instantaneous loss power of $\mathrm{M}_{2}$ when it turns on and turns off are shown in Fig. 14. The oscillation is attenuated and the circuit works normally. $p_{\mathrm{s}}$ is the power loss when $\mathrm{M}_{2}$ turns on or turns off. The definition of $p_{\mathrm{s}}$ also applies to the following formulas and figures.

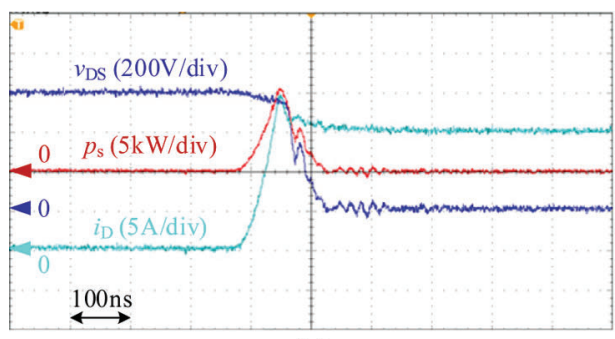

(a)

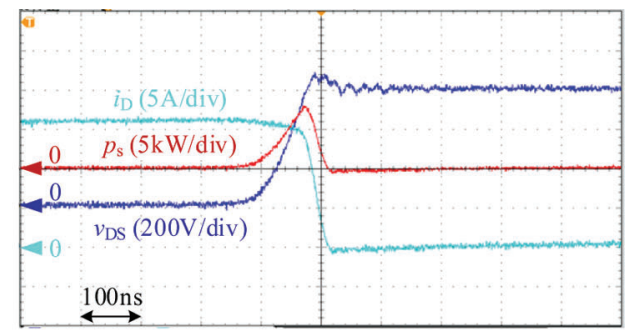

(b)

Figure 14: The drain-source voltage, the drain current and the instantaneous loss power of $\mathrm{M}_{2}$ at the DC bus-bar voltage of $600 \mathrm{~V}$ and the driver resistance of $206.5 \Omega$ : (a) when it turns on, and (b) when it turns off 
In this situation, the turn-on and turn-off time of the SiC MOSFET is slightly more than $100 \mathrm{~ns}$. The switching loss can be obtained by

$E_{\text {on }}=\int_{t_{1}}^{t_{2}} p_{s} d t$

$E_{\text {off }}=\int_{t_{3}}^{t_{4}} p_{s} d t$

where $E_{\text {on }}$ is the turn-on loss; $E_{\text {off }}$ is the turn-off loss; $t_{1}$ is the moment when the drain current begins to rise; $t_{2}$ is the moment when the drain-source voltage finishes falling; $t_{3}$ is the moment when the drain-source voltage begins to rise; $t_{4}$ is the moment when the drain current finishes falling. Through calculation, the turn-on loss of $\mathrm{M}_{2}$ is $600 \mu \mathrm{J}$, and the turn-off loss is $400 \mu \mathrm{J}$. Note that although the switching time and the switching loss of the $\mathrm{SiC}$ MOSFET at this time do not reach the minimum value that can be achieved, the SiC MOSFET still has the advantage because the switching time and the switching loss are much smaller compared with those of the Si IGBT at the same power level.

The switching loss of the SiC MOSFET is related to the charging and the discharging time of the input capacitance of the $\mathrm{SiC}$ MOSFET. The longer the charging and discharging time is, the greater the loss is. The charging and discharging time depends on the gate turn-on resistance and gate turn-off resistance respectively. The turn-on loss and the turn-off loss can be regulated respectively by adjusting the gate turn-on resistance and the gate turn-off resistance. Since there is no shoot-through problem during the turn-off process of the SiC MOSFET, the oscillation is relatively small, which is also verified by the above experiments. Therefore, in order to reduce the switching loss of the SiC MOSFET, the gate turnoff resistance can be appropriately reduced to speed up the turn-off time, which will not lead to serious oscillation at the turn-off process of the $\mathrm{SiC}$ MOSFET.

When the turn-on gate resistance is $206.5 \Omega$ and the turn-off gate resistance is $16 \Omega, 24.7 \Omega$ and $106.5 \Omega$ respectively, the switching waveforms are shown in Fig. 15. In Fig. 15, the dark blue waveform is the drainsource voltage of $\mathrm{M}_{2}$, the light blue waveform is the drain current of $\mathrm{M}_{2}$, and the red waveform is the instantaneous loss power of $\mathrm{M}_{2}$.

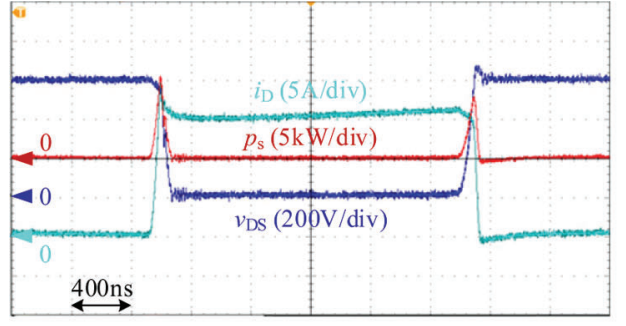

(a)

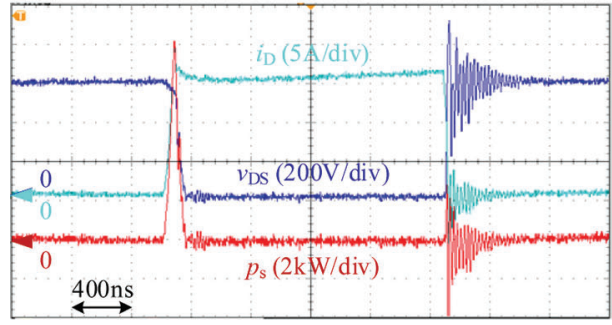

(c)

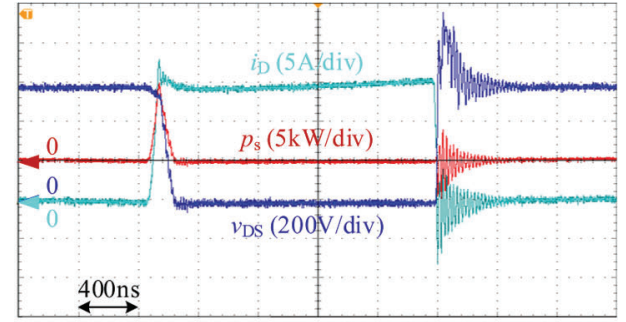

(b)

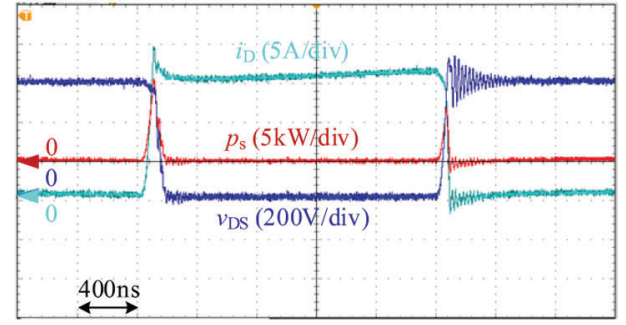

(d)

Figure 15: Switching waveforms of $\mathrm{M}_{2}$ at different turn-off gate resistance: (a) $206.5 \Omega$, (b) $16 \Omega$, (c) 24.7 $\Omega$, and (d) $106.5 \Omega$ 
When the turn-off gate resistance is $16 \Omega$ or $24.7 \Omega$, the turn-off oscillation is too large, affecting the normal work of the circuit. When the turn-off gate resistance is $106.5 \Omega$, the turn-off oscillation is moderate. The detailed switching processes of $\mathrm{M}_{2}$ are shown in Fig. 16. Through calculation, the turn-on loss is $600 \mu \mathrm{J}$, and the turn-off loss is $320 \mu \mathrm{J}$. The turn-on loss is the same as the above situation, and the turn-off loss is only the $80 \%$ of above situation, which means that decrease the turn-off gate resistance can reduce the turn-off loss on the premise of the less turn-on oscillation. Adopting this method, both the oscillation and the turn-off loss can be reduced to some extent, but the loss at the turn-on process cannot be reduced due to the big turn-on resistance.

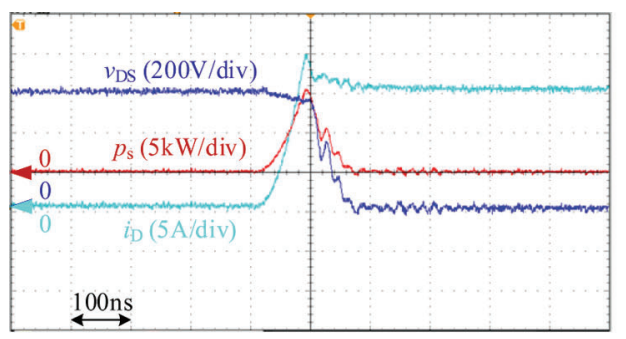

(a)

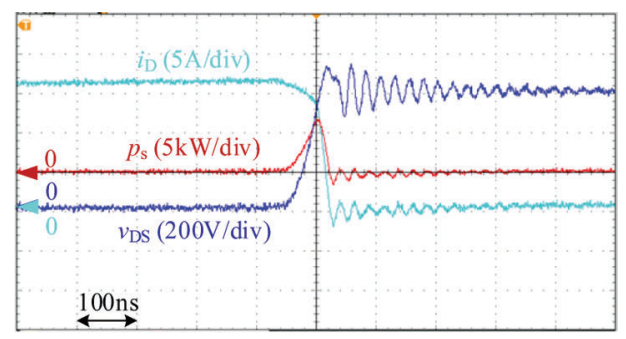

(b)

Figure 16: Detailed switching processes of $\mathrm{M}_{2}$ when the turn-on gate resistance is $206.5 \Omega$ and the turn off gate resistance is $106.5 \Omega$ : (a) turn-on waveforms, and (b) turn-off waveforms

Note that a gate resistance value around $200 \Omega$ does not exist in practical applications because of the huge switching losses. The experiment of $206.5 \Omega$ gate resistance in this paper is also set up to prove this point. In order to replace the $\mathrm{Si}$ IGBT with the $\mathrm{SiC}$ MOSFET to upgrade the converter without redesigning the circuit hardware structure, the first problem to be solved is the severe oscillation caused by the huge parasitic inductance. In order to reduce the severe oscillation, making the circuit work normally under the rated voltage, the traditional approach is to increase the gate resistance, but through the test of this paper, the resistance need to be increased to a very large value, such as $206.5 \Omega$. This is not practical, so this paper proposes a new method that inserts a ferrite bead in the main circuit to suppress the severe oscillation, which will be discussed below.

\subsection{Oscillation Suppression Method of Inserting the Ferrite Bead in the Circuit}

Inhibiting the circuit oscillation by increasing the driver resistance will inevitably increase the switching loss of the circuit, which will greatly reduce the advantage brought by the SiC MOSFET, so it is not a desirable solution. According to the above analysis, the ferrite bead can have a good damping effect on the high frequency electric quantity and it has no obvious effect on the switching speed of the $\mathrm{SiC}$ MOSFET. Thus, the ferrite bead can be used to reduce the oscillation without increasing the switching loss.

In the experiment, the ferrite beads are placed in the position shown in Fig. 8. Since the ferrite bead mainly play a large role in the oscillation during turn-on process of the $\mathrm{M}_{2}$, in order to reduce the turn-on loss, the turn-on gate resistance and the turn-off gate resistance in the experiment are $26.5 \Omega$ and $106.5 \Omega$ respectively. The switching waveforms of $\mathrm{M}_{2}$ at this time are shown in Fig. 17.

It can be found from Fig. 17 that the oscillation during the turn-on process is almost completely eliminated even if the turn-on resistance is only $20 \Omega$, which does not work properly at all before the ferrite bead is inserted. According to the calculation, the turn-on loss of $\mathrm{M}_{2}$ is $280 \mu \mathrm{J}$, and the turn-off loss of $\mathrm{M}_{2}$ is $400 \mu \mathrm{J}$. Compared to the above switching loss without the ferrite bead when the turn-on gate resistance is $206.5 \Omega$ and turn-off gate resistance is $106.5 \Omega$, the turn-on loss is decreased by $320 \mu \mathrm{J}$ $(53 \%)$ and the turn-off loss is increased by $80 \mu \mathrm{J}(25 \%)$. The increase of the turn-off loss is due to the 
effect of the ferrite bead on the turn-off speed. However, since the ferrite bead can reduce the allowed turn-on gate resistance, the total switching loss is still reduced by $240 \mu \mathrm{J}(26 \%)$ after the ferrite bead is adopted on the premise of ensuring a small oscillation in the circuit. Thus, the ferrite bead ensures that the oscillation and the switching loss are both small when the Si IGBT in the conventional power electronics equipment is replaced by the SiC MOSFET.

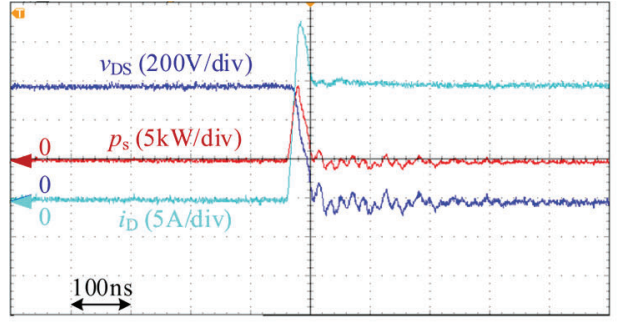

(a)

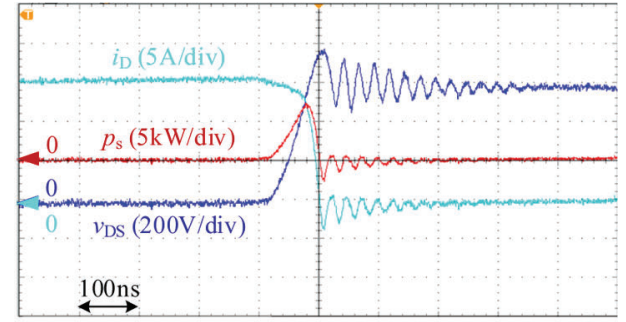

(b)

Figure 17: Switching waveforms of $\mathrm{M}_{2}$ after the ferrite bead inserted: (a) turn-on waveforms; (b) turn-off waveforms

\section{Conclusions}

Due to the influence of parasitic parameters in the traditional power electronics converter suitable for the Si IGBT, a serious circuit oscillation problem will occur when the Si IGBT is directly replaced by the $\mathrm{SiC}$ MOSFET. In this paper, the oscillation model with the adoption of the SiC MOSFET is established in the double-pulse test circuit and compared with the oscillation model after the ferrite bead being inserted in the main circuit. It is found that the ferrite bead has a good damping effect on the high-frequency oscillation and can relieve the cross-talk problem in the circuit. Through experiments, the three methods to suppress the oscillation are compared: Adopting the large driver resistance, the large turn-on gate resistance combined with the smaller turn-off gate resistance, and the ferrite bead combined with the smaller driver resistance. The last method can not only effectively suppress the oscillation, but also give full play to the advantage of small switching loss brought by the SiC MOSFET. Thus, to replace the $\mathrm{Si}$ IGBT in the traditional converter with the SiC MOSFET without redesigning the circuit, the ferrite bead can be inserted in the main circuit.

Funding Statement: This work was supported in part by the Natural Science Foundation of Jiangsu Province under Grant BK20200969 (L. Z., URL: http://std.jiangsu.gov.cn/), and in part by Nantong Science and Technology Plan Project under Grant JC2019095 (L. R., URL: http://kjj.nantong.gov.cn/)

Conflicts of Interest: The authors declare that they have no conflicts of interest to report regarding the present study.

\section{References}

1. Chen, Z., Yao, Y., Boroyevich, D., Ngo, K. D. T., Mattavelli, P. et al. (2014). A 1200-V, 60-A SiC MOSFET multichip phase-leg module for high-temperature, high-frequency applications. IEEE Transactions on Power Electronics, 29(5), 2307-2320. DOI 10.1109/TPEL.2013.2283245.

2. Zhong, X., Wu, X., Zhou, W., Sheng, K. (2014). An all-SiC high-frequency boost DC-DC converter operating at $320^{\circ} \mathrm{C}$ junction temperature. IEEE Transactions on Power Electronics, 29(10), 5091-5096. DOI 10.1109/ TPEL.2014.2311800.

3. Hazra, S., De, A., Cheng, L., Palmour, J., Schupbach, M. et al. (2016). High switching performance of 1700 V, $50 \mathrm{~A} \mathrm{SiC} \mathrm{power} \mathrm{MOSFET} \mathrm{over} \mathrm{Si} \mathrm{IGBT/BiMOSFET} \mathrm{for} \mathrm{advanced} \mathrm{power} \mathrm{conversion} \mathrm{applications.} \mathrm{IEEE}$ Transactions on Power Electronics, 31(7), 4742-4754. DOI 10.1109/TPEL.2015.2432012. 
4. Palmour, J. W., Cheng, L., Pala, V., Brunt, E. V., Lichtenwalner, D. J. et al. (2014). Silicon carbide power MOSFETs: Breakthrough performance from $900 \mathrm{~V}$ up to $15 \mathrm{kV}$. 2014 IEEE 26th International Symposium on Power Semiconductor Devices \& IC's (ISPSD), pp. 79-82. Waikoloa, HI, USA.

5. Zhang, H., Tolbert, L. M. (2011). Efficiency impact of silicon carbide power electronics for modern wind turbine full scale frequency converter. IEEE Transactions on Industrial Electronics, 58(1), 21-28. DOI 10.1109/ TIE.2010.2048292.

6. Whitaker, B., Barkley, A., Cole, Z., Passmore, B., Martin, D. et al. (2014). A high-density, high-efficiency, isolated on-board vehicle battery charger utilizing silicon carbide power devices. IEEE Transactions on Power Electronics, 29(5), 2606-2617. DOI 10.1109/TPEL.2013.2279950.

7. Bertelshofer, T., Horff, R., Maerz, A. (2016). A performance comparison of a $650 \mathrm{~V}$ Si IGBT and SiC MOSFET inverter under automotive conditions. Proceeding of PCIM, pp. 653-660, Nuremberg, Germany.

8. Doustad, D., Ameziani, M., Lhotellier, D., Lefebvre, S., Petit, M. (2017). Estimation of the losses in Si and SiC power modules for automotive applications. Proceeding of PCIM, pp. 1814-1821, Nuremberg, Germany.

9. Zeng, C., Li, Z., Yuan, F., Jiang, X., He, Z. et al. (2018). Comparison of SiC synchronous rectification and schottky diode in voltage source inverters. 2018 IEEE Energy Conversion Congress and Exposition, pp. 1903-1906. Portland, OR, USA.

10. Zhang, L., Yuan, X., Zhang, J., Wu, X., Zhang, Y. et al. (2019). Modeling and implementation of optimal asymmetric variable dead-time setting for $\mathrm{SiC}$ MOSFET-based three-phase two-level inverters. IEEE Transactions on Power Electronics, 34(12), 11645-11660. DOI 10.1109/TPEL.2019.2905882.

11. Pitlini, R., Zhang, Z., Andersen, M. (2013). Switching performance evaluation of commercial SiC power devices ( $\mathrm{SiC}$ JFET and SiC MOSFET) in relation to the gate driver complexity. Proceeding of IEEE ECCE Asia Downunder, pp. 233-239, Melbourne, VIC, Australia.

12. Cree/Wolfspeed (2015). Design considerations for designing with Cree $\mathrm{SiC}$ modules part 1. Understanding the effects of parasitic inductance. https://www.wolfspeed.com/downloads/dl/file/id/180/product/0/design_<br $></$ br $>$ considerations for designing with cree sic modules part 1 understanding the effects of parasitic inductance.pdf.

13. Ke, J., Zhao, Z., Xie, Z., Wei, C., Sun, P. (2017). Modeling and simulation of SiC MOSFET turn-off oscillation under influence of parasitic parameter. Proceeding of 2017 Sixth Asia-Pacific Conference on Antennas and Propagation, Xi'an, China.

14. Cree/Wolfspeed (2015). Design considerations for designing with Cree SiC modules part 2. Techniques for minimizing parasitic inductance. https://www.wolfspeed.com/downloads/dl/file/id/181/product/0/ design_considerations_ for designing with cree sic modules part 2 techniques for minimizing parasitic inductance.pdf.

15. Li, H., Munk-Nielsen, S. (2014). Challenges in switching SiC MOSFET without ringing. Proceeding of Pcim Europe, International Exhibition \& Conference for Power Electronics. VDE, pp. 1-6, Nuremberg, Germany.

16. Huang, H., Yang, X., Wen, Y., Long, Z. (2016). A switching ringing suppression scheme of SiC MOSFET by active gate drive. Proceeding of 2016 IEEE 8th International Power Electronics and Motion Control Conference (IPEMC 2016-ECCE Asia), pp. 285-291, Asia Hefei, China.

17. Kim, J., Shin, D., Sul, S. (2018). A damping scheme for switching ringing of full SiC MOSFET by air core PCB circuit. IEEE Transactions on Power Electronics, 33(6), 4605-4615. DOI 10.1109/TPEL.2017.2731861.

18. Chen, Z., Amaro, I. (2012). Optimizing low side gate resistance for damping phase node ringing of synchronous buck converter. Proceeding of IEEE Energy Conversion Congress \& Exposition, pp. 1827-1832, Raleigh, NC, USA.

19. Yatsugi, K., Nomura, K., Hattori, Y. (2018). Analytical technique for designing an RC snubber circuit for ringing suppression in a phase-leg configuration. IEEE Transactions on Power Electronics, 33(6), 4736-4745. DOI 10.1109/TPEL.2017.2731421.

20. Chuai, G., Wang, J., Yuan, X., Lai, B., Zhang, Q. (2019). Design and parasitic parameters analysis of a LCC resonant inverter using SiC MOSFET. Proceeding of 2019 22nd International Conference on Electrical Machines and Systems, Harbin, China.

21. Liu, B., Li, W., Meng, D., Diao, L., Ma, Y. et al. (2020). Low-stray inductance optimized design for power circuit of SiC-MOSFET-based inverter. IEEE Access, 8, 20749-20758. DOI 10.1109/ACCESS.2020.2964687. 
22. Yatsugi, K., Oishi, K., Iizuka, H. (2021). Ringing suppression of SiC MOSFET using a strongly coupled external resonator through analogy with passive PT-symmetry. IEEE Transactions on Power Electronics, 36(3), 29642970. DOI 10.1109/TPEL.2020.3013399.

23. Jahdi, S., Alatise, O., Gonzalez, J. A. O., Bonyadi, R., Ran, L. et al. (2016). Temperature and switching rate dependence of crosstalk in Si-IGBT and $\mathrm{SiC}$ power modules. IEEE Transactions on Industrial Electronics, 63(2), 849-863. DOI 10.1109/TIE.2015.2491880.

24. Zhang, L., Yuan, X., Wu, X., Shi, C., Zhang, J. et al. (2019). Performance evaluation of high-power SiC MOSFET modules in comparison to Si IGBT modules. IEEE Transactions on Power Electronics, 34(2), 1181-1196. DOI 10.1109/TPEL.2018.2834345.

25. Zhang, Z., Zhang, W., Wang, F., Tolbert, L. M., Blalock, B. J. (2012). Analysis of the switching speed limitation of wide band-gap devices in a phase-leg configuration. Proceeding of Energy Conversion Congress \& Exposition, pp. 3950-3955, Raleigh, NC, USA.

26. Xu, S., Sun, W., Sun, D. (2010). Analysis and design optimization of brushless DC motor's driving circuit considering the $\mathrm{Cdv} / \mathrm{dt}$ induced Effect. Proceeding of 2010 IEEE Energy Conversion Congress and Exposition, pp. 2091-2095, Atlanta, GA, USA.

27. Li, H., Jiang, Y., Qiu, Z., Wang, Y., Ding, Y. (2021). A predictive algorithm for crosstalk peaks of SiC MOSFET by considering the nonlinearity of gate-drain capacitance. IEEE Transactions on Power Electronics, 36(3), 28232834. DOI 10.1109/TPEL.2020.3016155.

28. Yin, S., Tseng, K. J., Tong, C. F., Simanjorang, R., Gajanayake, C. J. et al. (2015). Gate driver optimization to mitigate shoot-through in high-speed switching $\mathrm{SiC}$ half bridge module. IEEE 11th International Conference on Power Electronics and Drive Systems, pp. 484-491, Sydney, NSW, Australia.

29. Zhang, Z., Wang, F., Tolbert, L. M., Blalock, B. J. (2014). Active gate driver for crosstalk suppression of SiC devices in a phase-leg configuration. IEEE Transactions on Power Electronics, 29(4), 1986-1997. DOI 10.1109/TPEL.2013.2268058.

30. Tang, H., Chung, H. S., Fan, J. W., Yeung, R. S., Lau, R. W. (2020). Passive resonant level shifter for suppression of crosstalk effect and reduction of body diode loss of SiC MOSFETs in bridge legs. IEEE Transactions on Power Electronics, 35(7), 7204-7225. DOI 10.1109/TPEL.2019.2957985.

31. Li, C., Lu, Z., Chen, Y., Li, C., Luo, H. et al. (2020). High off-state impedance gate driver of SiC MOSFETs for crosstalk voltage elimination considering common-source inductance. IEEE Transactions on Power Electronics, 35(3), 2999-3011. DOI 10.1109/TPEL.2019.2932263.

32. Zhang, B., Xie, S., Xu, J., Qian, Q., Zhang, Z. et al. (2017). A magnetic coupling based gate driver for crosstalk suppression of SiC MOSFETs. IEEE Transactions on Industrial Electronics, 64(11), 9052-9063. DOI 10.1109/ TIE.2017.2736500. 